\title{
Relationship between Azathioprine metabolites and therapeutic efficacy in Chinese patients with neuromyelitis optica spectrum disorders
}

Xindi $\mathrm{Li}^{1,2}$, Shenghui Mei ${ }^{3}$, Xiaoqing Gong ${ }^{1,2}$, Heng Zhou ${ }^{1,2}, \mathrm{Li} \mathrm{Yang}^{3}$, Anna Zhou ${ }^{1,2}$, Yonghong Liu ${ }^{1,2}$, Xingang Li ${ }^{3}$, Zhigang Zhao ${ }^{3^{*}}$ and Xinghu Zhang ${ }^{1,2^{*}}$

\begin{abstract}
Background: Neuromyelitis optica spectrum disorders (NMOSD) are demyelinating autoimmune diseases in the central nervous system (CNS) that are characterized by a high relapse rate and the presence of anti-aquaporin 4 antibodies (AQP4-lgG) in the serum. Azathioprine (AZA) is a first-line immunomodulatory drug that is widely used for the treatment of patients with NMOSD. However, the efficacy and safety of AZA vary in different individuals.

Method: Thirty-two patients with NMOSD who regularly took AZA were enrolled in the study at Beijing Tiantan Hospital, Capital Medical University. The efficacy of AZA was evaluated using the expanded disability status scale (EDSS) and the annual relapse rate (ARR). The erythrocyte concentrations of AZA metabolites were detected using an LC-MS/MS method.
\end{abstract}

Results: The erythrocyte concentrations of 6-thioguanine nucleotides (6-TGNs) and 6-methylmercaptopurine nucleotides (6-MMPNs) were $202.03 \pm 63.35 \mathrm{pmol} / 8^{*} 10^{8} \mathrm{RBC}$ and $1618.90 \pm 1607.06 \mathrm{pmol} / 8^{*} 10^{8} \mathrm{RBC}$, respectively. After the patients had received AZA therapy for more than one year, the EDSS score decreased from $5.21 \pm 0.24$ to $2.57 \pm 0.33(p<0.0001)$, and the ARR decreased from $1.41 \pm 0.23$ to $0.36 \pm 0.09(p<0.0001)$. The 6-TGN and 6-MMPN levels were significantly different between the non-relapsed and relapsed groups ( $p<0.0001, p=0.006$, respectively). A higher ARR was significantly correlated with higher erythrocyte concentrations of 6-TGNs $(p<0.0001)$ and 6-MMPNs $(p=0.004)$.

Conclusion: AZA can reduce the EDSS score and ARR in NMOSD patients. Additionally, the efficacy of AZA is significantly related to the erythrocyte concentrations of 6-TGNs and 6-MMPNs. Within the safe upper limits, a higher concentration of 6-TGNs is associated with better efficacy of AZA.

Trial registration number: ISRCTN16551495, retrospectively registered on May 22, 2017.

Keywords: Neuromyelitis optica spectrum disorders (NMOSD), Azathioprine (AZA), Thiopurine S-methyltransferase (TPMT), 6-thioguanine nucleotides (6-TGNs), 6-methylmercaptopurine nucleotides (6-MMPNs)

\footnotetext{
*Correspondence: ttyyzzg1022@126.com; xhzhtiantan@hotmail.com ${ }^{3}$ Department of Pharmacy, Beijing Tiantan Hospital, Capital Medical University, 6 TiantanXili, Dongcheng District, Beijing 100050, People's Republic of China

${ }^{1}$ Neuroinfection and Neuroimmunology Center, Department of Neurology, Beijing Tiantan Hospital, Capital Medical University, 6 TiantanXili, Dongcheng District, Beijing 100050, People's Republic of China

Full list of author information is available at the end of the article
} 


\section{Background}

Neuromyelitis optica spectrum disorders (NMOSD) are demyelinating autoimmune diseases in the central nervous system (CNS) that are characterized by the presence of anti-aquaporin four antibodies (AQP4-IgG) in the serum [1]. The clinical disability progressively deteriorates, and the occurrence of relapses increases. Currently, azathioprine (AZA), which is a first-line immunomodulatory drug, is widely used for the prevention of relapse in NMOSD patients [2]. The efficacy of the AZA treatment in NMOSD patients has been mainly evaluated by expanded disability status scale (EDSS) values and the annual relapse rates (ARR). Elsone et al. reported that neurological function improved or remained stable in $78 \%$ of patients who received ASA therapy, with a mild reduction in the mean EDSS score from 5.5 to 4 in 103 NMOSD patients [3]. The ARR has been shown to decrease after treatment with AZA [4, 5]. In a Chinese retrospective study, 57.1\% of NMOSD patients were in relapse-free status [6]. However, the efficacy and safety of AZA vary in different individuals. Severe adverse reactions to AZA, such as leukopenia and liver dysfunction, may limit its use [7]. Therefore, the identification of indicators to guide the safe and effective use of AZA is very important.

The immunosuppressive effects of AZA are mainly due to its metabolites. AZA rapidly transforms into 6mercaptopurine (6-MP), which is further converted into thiopurine nucleotides (TPNs), including 6-thioguanine nucleotides (6-TGNs) and 6-methylmercaptopurine nucleotides (6-MMPNs), by a series of competitive enzymes [8]. 6-TGNs are produced by hypoxanthineguanine phosphoribosyltransferase (HGPRT), and 6MMPNs, which are the inactive by products of AZA, are formed by thiopurine-methyltransferase (TPMT) $[9,10]$. The intracellular accumulation of 6-TGNs and 6MMPNs can inhibit the biosynthesis of nucleic acids and prevent the proliferation of various lymphocytes [11]. The control of lymphocyte apoptosis is vital to the regulation of the immune system. Therefore, the immunosuppression effect of AZA was correlated with the accumulation of 6-TGNs and 6-MMPNs [12].

The AZA metabolites are mainly mediated by TPMT [13]. Allozymes encoded by certain TPMT mutant alleles displayed low TPMT activity that was undetectable after their expression in COS-1 cells [14]. TPMT $22(238 \mathrm{G}>\mathrm{C})$, TPMT*3A (460G > A and719A > G), TPMT*3B (460G > A) and TPMT:3C (719A > G) account for $80-95 \%$ of patients with low TPMT activity $[15,16]$. Pyrimidine and purine nucleosides, such as 6-TGNs and 6-MMPNs, are transported into the cells by the following two types of human nucleoside transporters: concentrative nucleoside transporters and equilibrative nucleoside transporters. These transporters are encoded by two gene families (i.e., SLC28 and $S L C 29)[17,18]$. Badagnani et al. reported that CNT3 (i.e., SLC28A3) played an important role in the mediation of the cellular entry of a variety of physiological nucleosides and synthetic anticancer nucleoside analog drugs [19]. SLC28A3 was also reported to have a significant influence on the transport of 6-MP [20]. The relationship between polymorphisms of $S L C 28 A 3$ and the erythrocyte concentrations of AZA metabolites in NMOSD has been demonstrated in our former study [21]. Based on the previous study, this study analyzed the relationship between the concentrations of AZA metabolites and the immunosuppressive efficacy of AZA to identify predictive biomarkers for efficacy evaluations of AZA in NMOSD patients.

\section{Methods}

Subjects

This study analyzed the clinical characteristics, AZA metabolites and genetic polymorphism of prospectively enrolled patients. The study was approved by the Ethics Committee of Beijing Tiantan Hospital Affiliated to Capital Medical University, Beijing, People's Republic of China (No. KY2015-031-02). Written informed consent was obtained from the patients or from their parents / legal guardians who were under 18 or from the close relatives whose participants had severe disability in the writing hand or the illiteracy. Forty-one patients with NMOSD were enrolled. All patients received steroids during the acute disease stage. The initial dosage of methylprednisolone was $1000 \mathrm{mg}$ for 3 days, which was tapered as follows: $500 \mathrm{mg}$ for 3 days, $250 \mathrm{mg}$ for 3 days and $120 \mathrm{mg}$ for 3 days. Then, oral prednisone (60 mg per day) was administered and slowly withdrawn within 12 weeks. AZA therapy was added at the beginning of the oral prednisone administration. The initial dosage of AZA was $50 \mathrm{mg}$ per day for the first 5 days. If no severe adverse reactions appeared, the dosage of AZA was increased to $100 \mathrm{mg}$ per day. Routine blood tests and hepatic and renal functions were monitored regularly (during the first month of the AZA intake, monitoring was performed once every week; during the 2nd month, monitoring was performed once every two weeks; during the 3rd month and thereafter, monitoring was performed once a month). The treatment was stopped if severe adverse reactions occurred. Nine patients withdrew from the study due to the appearance of severe adverse reactions. If relapse occurred, high-dose steroids were re-introduced and withdrawn as previously described.

The inclusion criteria in this study were as follows:

1. Met the International Consensus Diagnostic Criteria for Neuromyelitis Optica Spectrum Disorder 2015 [1].

2. Onset age: 12 to 80 years.

3. No previous exposure to any immunosuppressive agent. 
4. Did not undergo blood transfusion three months before sampling.

5. Received more than 12 months of AZA treatment, and the dose has not been changed within the previous 4 weeks to ensure a stable AZA metabolite profile.

The exclusion criteria were as follows:

1. Intolerance to the AZA treatment due to any severe adverse reaction, such as the leukocyte counts less than $4 \times 10^{9} / \mathrm{L}$, other severe cardiovascular disease or hepatopathy.

2. Planned or current pregnancy and/or breast feeding.

3. Other unsuitable characteristics as determined by the clinicians.

\section{Methods}

The disability was measured by the EDSS. The pretherapy EDSS was evaluated at the stable stage (more than one month after relapse), and the post-therapy EDSS was evaluated at the final visit (at least one year after the AZA treatment). The ARR was calculated according to the number of relapses per year. To remove the influence of the pretherapy ARR, we measure the ARR improvement as the pretherapy ARR minus the posttherapy ARR divided by the pretherapy ARR (the value of the ARR improvement was positive). A confirmed relapse was defined as the appearance of new neurological symptoms or worsening of preexisting symptoms that lasted for at least $24 \mathrm{~h}$ and was accompanied by an objective neurological change (worsening by 0.5 points on the EDSS or by $\geq 1.0$ points on the pyramidal sign, cerebella, brainstem, or visual functional system scores) in patients who had been neurologically stable or improving in the previous 30 days [6]. The relapsed group included patients who had a confirmed relapse after taking AZA. The non-relapsed group included patients who did not experience any relapse event during the followup duration. The AZA efficacy was evaluated based on the changes in the EDSS score and the ARR after the therapy.

CSF and serum samples for the immunological tests, including CSF protein, CSF IgG and AQP4-IgG, were routinely collected during the acute phase before any therapy was administered. Cell-based assays were used for the serum AQP4-IgG detection [22]. Blood samples $(5 \mathrm{~mL})$ were collected in vacuum tubes (containing ethylenediaminetetraacetic acid) after 30 days of regular AZA therapy (during the remission phase). After centrifugation at $5000 \times g$ for $10 \mathrm{~min}$, the plasma was removed, and the white blood cells were stored at $-80{ }^{\circ} \mathrm{C}$ for genotyping. The erythrocytes were washed twice with $2 \mathrm{ml}$ saline and centrifuged at $10000 \times g$ for $2 \mathrm{~min}$ to remove the supernatant, which was subsequently stored at
$-80{ }^{\circ} \mathrm{C}$ to detect the concentrations of erythrocyte 6TGNs and 6-MMPNs using our previously reported method (high-performance liquid chromatographic tandem mass spectrometry) [21]. The measurements of TPNs were divided by the body mass index, total daily dose, and mean corpuscular volume to remove the influence of these variables.

\section{Statistical analysis}

SPSS (version 17, SPSS Inc., Chicago, IL, USA) was used for statistical analysis. The chi-square test was used to compare the ARR values (before and after the treatment) and the serum AQP4-IgG concentrations. Nonparametric independent-sample $k-s$ test was used to analyze the differences in the EDSS scores. Independent-samples $t$-test was used to analyze the differences in the other related variables. The relationships between the concentrations of the AZA metabolites and ARR improvement were evaluated by 2-tailed Spearman rank correlation and multiple linear-regression, which was adjusted for onset age, disease duration, therapy duration, pretherapy and post-therapy EDSS scores, and laboratory tests. A two-tailed $p$-value $<0.05$ was considered statistically significant.

\section{Results}

\section{Characteristics of NMOSD patients treated with AZA}

All 32 NMOSD patients (30 females and 2 males) regularly received AZA therapy. The average onset age was 33.28 years (ranged from 18 to $62, \mathrm{SD}=12.77$ ). The average duration of the disease was 85.56 months (ranged from 19 to $266, \mathrm{SD}=68.18$ ), and the average duration of AZA therapy was 21.50 months (ranged from 12 to 40, SD =6.19). In total, $21(65.6 \%)$ patients presented with optic neuritis, and 29 (90.6\%) patients presented with myelitis. The mean concentration of the CSF protein was $40.41 \pm 32.67 \mathrm{mg} / \mathrm{dl}$, and the mean CSF IgG index was $0.53 \pm 0.10$. Serum anti-AQP4 antibodies were positively detected in $14(43.75 \%)$ patients. The concentrations of erythrocyte 6-TGNs were within the safe upper limits $\left(6\right.$-TGN $\left.<450 \mathrm{pmol} / 8 \times 10^{8} \mathrm{RBC}\right)$, but a few patients had erythrocyte 6-MMPN concentrations above the safe upper limits $\left(5700 \mathrm{pmol} / 8 \times 10^{8} \mathrm{RBC}\right)$ [23]. The average 6-TGNs concentration was $202.03 \mathrm{pmol} / 8^{*} 10^{8} \mathrm{RBC}$ (ranged from 83.441 to 319.71 , $\mathrm{SD}=63.35$ ), and the average 6-MMPN concentration was $1618.90 \mathrm{pmol} / 8^{*} 10^{8} \mathrm{RBC}$ (ranged from 235.04 to 7498.89, SD $=1607.06)$. The mean EDSS score decreased from $5.21 \pm 0.24$ to $2.57 \pm 0.33(p<0.0001)$, and the mean ARR decreased from $1.41 \pm 0.23$ to $0.36 \pm 0.09$ $(p<0.0001)$. Detailed information is provided in Table 1 . The individual relapse events within 10 years before AZA therapy and 2 years after AZA therapy are shown in Fig. 1, and two patients (No. 6 and No. 14) had worse ARRs after AZA therapy. 
Table 1 The general characteristics of NMOSD patients

\begin{tabular}{|c|c|}
\hline Variable & Patients $(n=32)$ \\
\hline Mean Age of onset, year & $33.28(15-62)$ \\
\hline \multicolumn{2}{|l|}{$\operatorname{Sex}(n \%)$} \\
\hline Female & 30 (93.75\%) \\
\hline Male & $2(6.25 \%)$ \\
\hline Mean BMI & $24.17(17.57-31.65)$ \\
\hline Mean Disease duration, months & $85.56(19-266)$ \\
\hline Mean Duration of AZA therapy, months & $21.50(12-40)$ \\
\hline \multicolumn{2}{|l|}{ Clinical symptoms at onset (n\%) } \\
\hline Optic neuritis & $21(65.6 \%)$ \\
\hline Bilateral Optic neuritis & $17(53.13 \%)$ \\
\hline Myelitis & $29(90.6 \%)$ \\
\hline Optic neuritis and Myelitis & $19(59.3 \%)$ \\
\hline Mean Pre-therapy EDSS score & $5.21 \pm 0.24(2.5-8)$ \\
\hline Mean Post- therapy EDSS score & $2.57 \pm 0.33(0-7)$ \\
\hline Mean Concentration of CSF protein, mg/dl & $40.41 \pm 32.67(12-184)$ \\
\hline Mean Concentration of CSF lgG, mg/ml & $0.04 \pm 0.03(0.01-0.19)$ \\
\hline CSF lgG index (n\%) & $0.53 \pm 0.10(0.39-0.83)$ \\
\hline Serum anti-AQP4 antibodies (n\%) & $14(43.75 \%)$ \\
\hline $\begin{array}{l}\text { Mean Concentration of } 6-\mathrm{TGNs} \text {, } \\
\mathrm{pmol} / 8 \times 10^{8} \mathrm{RBC}\end{array}$ & 202.03 \\
\hline $\begin{array}{l}\text { Mean Concentration of MMPNs, } \\
\mathrm{pmol} / 8 \times 10^{8} \mathrm{RBC}\end{array}$ & 1618.90 \\
\hline Relapse, n\% & $12 / 32(37.5 \%)$ \\
\hline Pre- therapy ARR & $1.42 \pm 0.23(0.2-3.5)$ \\
\hline Post- therapy ARR & $0.36 \pm 0.09(0-1.72)$ \\
\hline
\end{tabular}

NMOSD Neuromyelitisoptica spectrum disorders, AZA Azathioprine, AQP4 antiaquaporin 4, BMI Body mass index, ARR Annual relapse rate, EDSS Expanded disability status scale, CSF cerebrospinal fluid, IgG Immunoglobulin G

The differences in the related variables between the nonrelapsed and relapsed groups

Except for the EDSS score, other variables were normally distributed with a homogeneity of variance. The mean values of the normalized 6-TGN levels (1.12 vs. $0.70, p<0.0001$ ) and 6-MMPN levels (10.60 vs. 3.62, $p=0.006)$ were significantly different between the nonrelapsed and relapsed groups (See Table 2). No significant differences were observed in the other variables.

\section{Relationships between the post-therapy ARR and the erythrocyte concentrations of 6-TGNs and 6-MMPNs}

The normalized erythrocyte concentrations of 6 -TGNs and 6-MMPNs followed a normal distribution. However, ARR improvement did not follow a normal distribution. Higher ARR improvements were significantly correlated with higher erythrocyte concentrations of both 6-TGNs (correlation coefficient $(\mathrm{R})=0.679, p<0.0001$ ) and 6MMPNs ( $R=0.493, p=0.004)$ (See Table 3 and Fig. 2).
The ARR improvement (in log) was normally distributed. According to the multiple linear-regression, the ARR improvement (in log) was significantly influenced by the erythrocyte concentrations of 6-TGNs (adjusted R square $=0.206$, constant $=0.417$, standardized coefficients beta $=-0.489, p=0.013)$. The remaining variables, including the erythrocyte concentration of 6-MMPNs, onset age, disease duration, treatment duration, pretherapy and posttherapy EDSS scores, mean CSF protein, mean concentration of CSF IgG and the CSF IgG index, were excluded from the analysis due to underpowered statistical significance $(p>0.05)$ (See Table 4$)$.

\section{Discussion}

The AZA treatment had a good efficacy in the NMOSD patients, and dramatic reductions in the EDSS scores (from 5.21 to 2.57, $p<0.0001$ ) and ARR values (from 1.41 to $0.36, p<0.0001$ ) were observed. These results may be partially due to the concomitant use of corticosteroids, which was reported in the literature $[6,24]$. The reduced ARR in our study was consistent with two large cohort studies in which the ARR decreased from 2.20 to 0.89 $(p<0.0001)$ and from 1.5 to $0(p<0.00005)[3,5]$.

Elsone et al. posed an interesting question of whether the apparent reduction in relapses was simply a 'regression to the mean', reflecting the natural course of the illness, or an immune-suppression effect of the AZA treatment [3]. In our study, no significant differences were observed in the pretherapy EDSS scores or onset of immunological status, including the CSF protein, CSF IgG index and serum AQP4-IgGs, between the relapsed and non-relapsed groups, and none of above parameters were correlated with the ARR improvement, which indicated homogeneity in individual disease activities. Therefore, we explored the relationships between the concentrations of the AZA metabolites and the posttherapy ARR. The mean normalized 6-TGNs levels $(1.12$ vs. $0.70, p<0.0001$ ) and mean normalized 6-MMPNs levels $(10.60$ vs. $3.62, p=0.006)$ were significantly higher in the non-relapsed patients than in the relapsed patients. A greater ARR improvement was correlated with higher erythrocyte concentrations of 6-TGNs $(R=0.679$, $p<0.0001)$ and 6-MMPNs $(R=0.493, p=0.004)$. 6TGNs are the main immunosuppressive compounds and have been proposed to induce the activation of nonspecific apoptotic pathways in proliferating lymphocytes by distorting DNA and weakening its repair system, which leads to apoptosis [25]. 6-MMPNs, which are strong inhibitors of purine de novo synthesis (PDNS), can block the proliferation of various types of lymphocyte lines [26]. In addition, the AZA metabolites can also induce a very specific apoptosis pathway in the CD4+ subset of CD28 co-stimulated $\mathrm{T}$ lymphocytes [12]. NMOSD is a CNS immunological disease that can be 


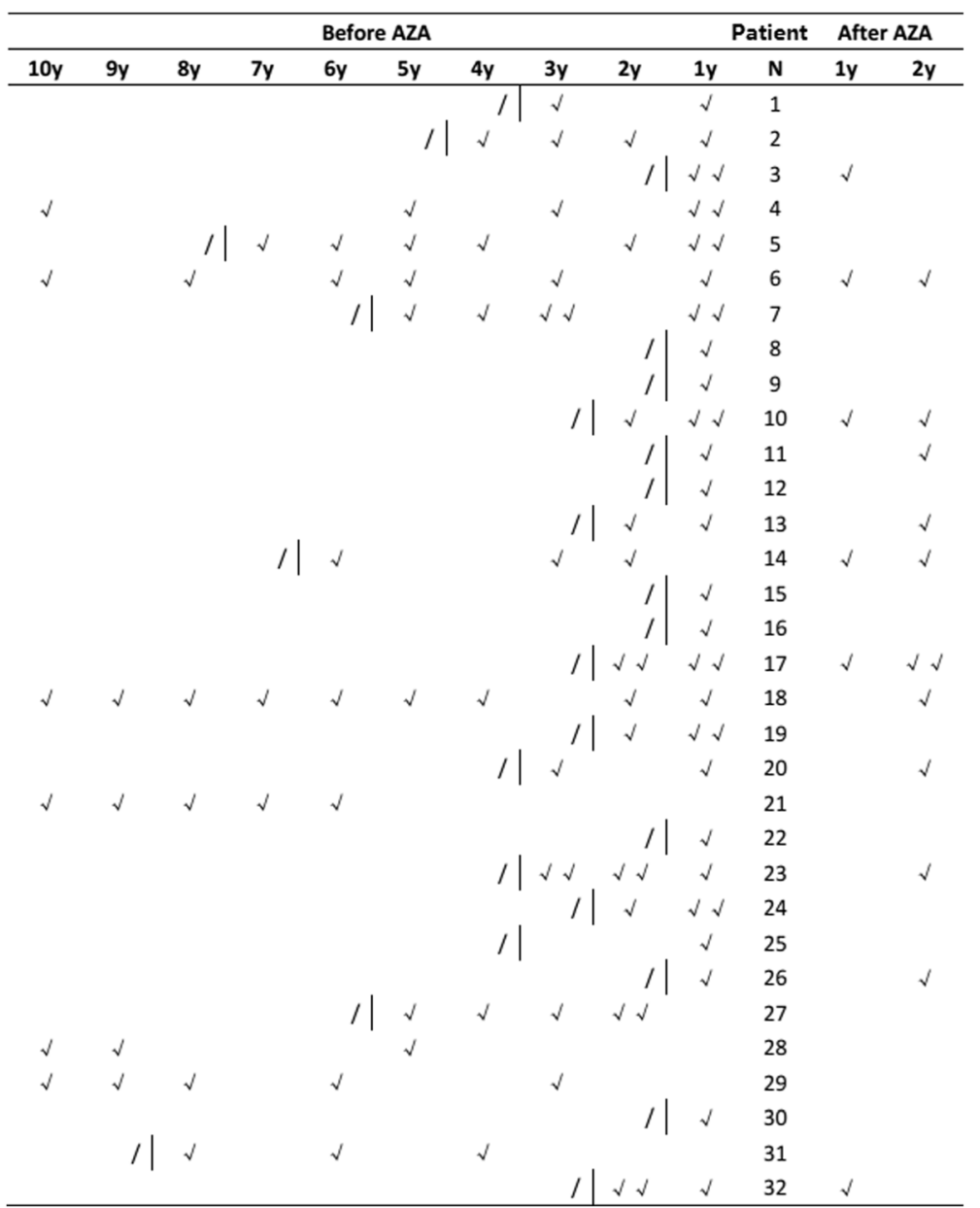

Fig. 1 The individual relapse times of each patient within 10 years before AZA therapy and 2 years after AZA therapy. "V" denotes the time of relapse. "I" denotes the beginning of AZA therapy. "/" indicates not available

Table 2 The difference of related variables between non-relapsed and relapsed groups

\begin{tabular}{|c|c|c|c|c|c|}
\hline Variable & Non-relapsed $(n=20)$ & Relapsed $(n=12)$ & Mean Difference & Std. Error Difference & $p$-value \\
\hline Age of onset, year & 33.35 & 33.16 & -0.183 & 4.740 & 0.969 \\
\hline Mean BMI & 23.89 & 24.63 & 0.741 & 1.415 & 0.604 \\
\hline Disease duration, months & 80.40 & 94.17 & 13.77 & 25.81 & 0.589 \\
\hline Duration of AZA therapy, months & 22.10 & 20.50 & -1.6 & 2.278 & 0.488 \\
\hline Mean CSF protein, mg/dl & 39.82 & 41.43 & 1.613 & 13.275 & 0.904 \\
\hline Mean Concentration of CSF lgG, mg/ml & 0.040 & 0.032 & -0.008 & 0.014 & 0.577 \\
\hline CSF IgG index (n\%) & 0.546 & 0.494 & -0.519 & 0.039 & 0.199 \\
\hline Serum anti-AQP4 antibodies (n\%) & $10(50 \%)$ & $4(33.3 \%)$ & / & / & 0.471 \\
\hline Pre-therapy EDSS score & 5.17 & 5.29 & / & / & 0.687 \\
\hline Post- therapy EDSS score & 2.43 & 2.83 & / & / & 0.893 \\
\hline Normalized concentration of 6-TGNs & 1.12 & 0.70 & -0.418 & 0.09 & $<0.0001^{*}$ \\
\hline Normalized concentration of 6-MMPNs & 10.60 & 3.62 & -6.983 & 2.32 & $0.006^{*}$ \\
\hline
\end{tabular}

NMOSD, AZA Azathioprine, BMI Body mass index, ARR Annual relapse rate, AQP4 anti-aquaporin 4, EDSS Expanded disability status scale, 6-TGN 6-thioguanine nucleotides, 6-MMPN 6-methylmercaptopurine nucleotides, CSF cerebrospinal fluid, IgG Immunoglobulin G. All two-tailed $P$-value $<0.05$ was considered statistically significant 
Table $\mathbf{3}$ The correlation between ARR improvements and normalized erythrocyte concentrations of AZA metabolites

\begin{tabular}{lll}
\hline Variable & $\mathrm{R}$ & $P$-value \\
\hline Normalized Concentration of 6-TGNs & 0.679 & $<0.0001^{*}$ \\
Normalized Concentration of MMPNs & 0.493 & $0.004^{*}$ \\
\hline
\end{tabular}

6-TGN 6-thioguanine nucleotides, 6-MMPN 6-methylmercaptopurine nucleotides, $R$ Spearman's correlation coefficient. All two-tailed $P$-value $<0.05$ was considered statistically significant

mediated by various lymphocytes, including Th17 lymphocytes, B lymphocytes and plasma-blasts [27]. After inhibiting the proliferation of lymphocytes by high levels of 6-TGNs and 6-MMPNs, the activation of immunoreactions decreased, and the mean ARR of the NMOSD patients subsequently declined.

The immunosuppressive effect of AZA was hypothesized to be achieved via a balanced contribution of proapoptotic (6-TGNs) and anti-metabolic (methylated ribonucleotides) pathways [8]. However, we found that the ARR improvement was only significantly influenced by the erythrocyte concentration of 6-TGNs (standardized coefficients beta $=-0.489, p=0.013$ ), which could be due to the pro-apoptosis pathways in which lymphocytes are induced by 6-TGNs, causing widespread damage to lymphocytes in the short-term treatment, and the lethality of the anti-metabolic effect increased with the gradual accumulation of 6-TGNs levels in the long-term treatment [8]. Therefore, we believe that 6 -TGNs may play a more important role in the immunosuppressive efficacy than previously thought, particularly during the early remission phase of NMOSD.

Furthermore, the internal variables that influence the erythrocyte concentrations of the AZA metabolites are unknown. The genetic polymorphisms of TPMT [28, 29] can affect TPMT activity, which directly regulates the formation of 6-MMPNs and subsequently influences the 6 -TGNs concentration. However, in some recently published articles, no correlation among TPMT activity, 6TGNs levels and 6-MMPNs levels was observed [30]. We found that rs10868138 (SLC28A3) was associated with a higher erythrocyte concentration of 6-TGNs, and rs12378361 (SLC28A3) was associated with a lower erythrocyte concentration of 6-TGNs in our former study [21]. The $S L C 28 A 3$ gene families correspond to human concentrative nucleoside transporters 3 (CNT3), which appears to be the best drug transporter because it can efficiently transport most of the pyrimidine and purine nucleoside analogs [31]. Our results indicated that the nucleoside transporter encoded by SLC28A3 (rs10868138) may participate in the metabolism of AZA by transporting 6-TGNs out of the cells, and SLC28A3 (rs12378361) may help transport 6-TGNs into the cells. In addition to its expression in the membranes of erythrocytes, CNT3 is also present in primary lymphocytes [32], monocytes, monocyte-derived macrophages, and monocyte-derived dendritic cell membranes [33]. Therefore, genetic polymorphisms of SLC28A3 (encoding CNT3) may influence the activity of NMOSD by altering the concentration of intracellular 6-TGNs in various immune cells. However, considering that $S L C 28 A 3$ is not the only factor that influences the concentration of intracellular 6-TGNs, the direct correlation between the polymorphisms of SLC28A3 and the outcomes of NMOSD must be investigation in further studies.

The disease activity in NMOSD is highly variable among individual patients, and some patients experience AZA efficacy after 6 months to 18 months in clinical practice. Some limitations were present in this study, including the use of patients from a single center, the small sample size and the short follow-up durations.
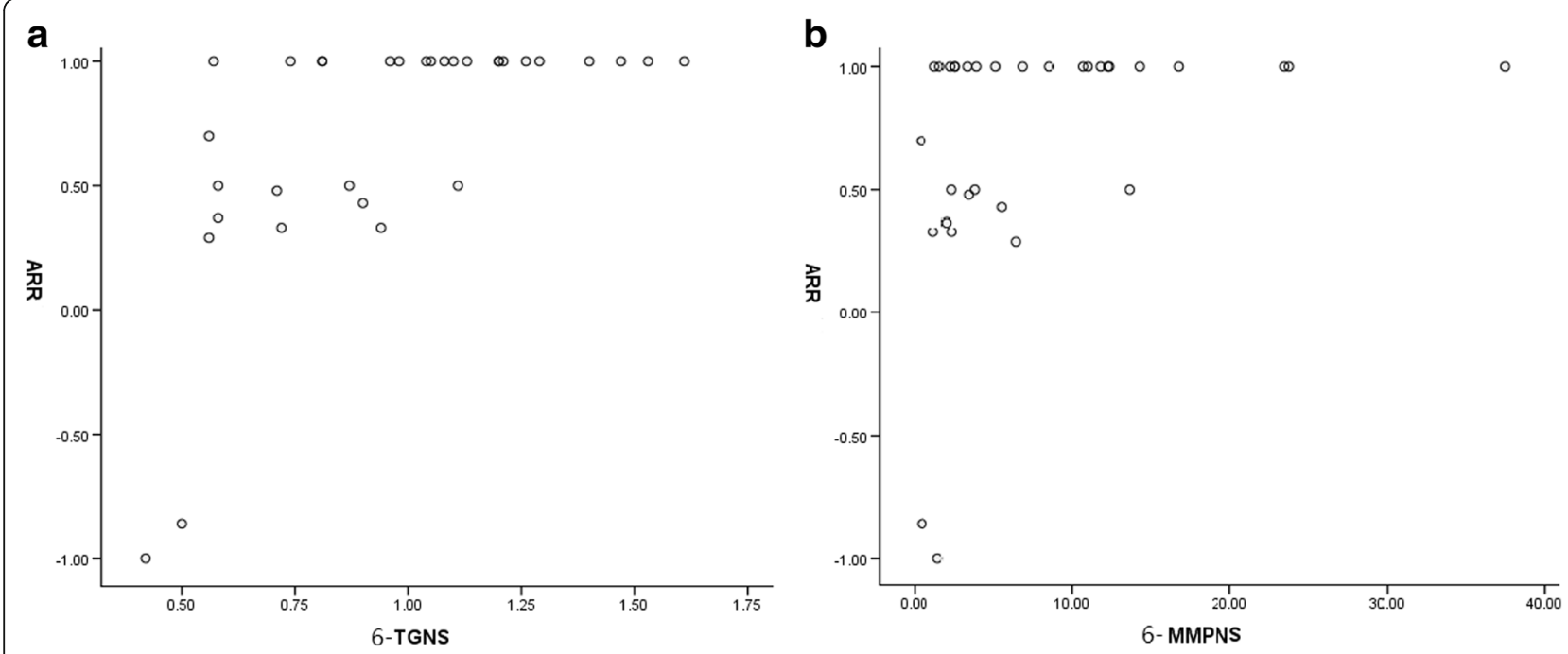

Fig. 2 a Plot of ARR improvements vs. 6-TGNs. b Plot of ARR improvements vs. 6-MMPNs 
Table 4 The linear- regression between multiple variables and ARR improvements (in log)

\begin{tabular}{|c|c|c|c|}
\hline Variable & Standardized coefficients- Beta & $\mathrm{T}$ & $P$-value \\
\hline Constant (SE) & $0.417(0.116)$ & 3.580 & 0.002 \\
\hline Normalized Concentration of 6-TGNs & -0.489 & -2.689 & $0.013^{*}$ \\
\hline \multicolumn{4}{|l|}{ Excluded variables } \\
\hline Age of onset, year & 0.156 & 0.853 & 0.403 \\
\hline Disease duration, months & 0.094 & 0.509 & 0.616 \\
\hline Treatment duration, months & -0.200 & -1.106 & 0.281 \\
\hline Pre-therapy EDSS score & 0.034 & 0.176 & 0.862 \\
\hline Post-therapy EDSS score & 0.050 & 0.266 & 0.793 \\
\hline Mean CSF protein, mg/dl & -0.038 & -0.198 & 0.845 \\
\hline Mean Concentration of CSF lgG, mg/ml & -0.158 & -0.855 & 0.402 \\
\hline CSF IgG index (n\%) & -0.287 & -1.636 & 0.116 \\
\hline Normalized Concentration of MMPNs & -0.150 & -0.751 & 0.461 \\
\hline
\end{tabular}

NMOSD, AZA Azathioprine, BMI Body mass index, ARR Annual relapse rate, EDSS Expanded disability status scale, 6-TGN 6-thioguanine nucleotides, 6-MMPN 6-methylmercaptopurine nucleotides. All $P$-value $<0.05$ was considered statistically significant

Our preliminary conclusions must be verified in further large cohort studies with long follow-up durations.

\section{Conclusion}

AZA treatment in NMOSD patients can dramatically reduce post-therapy EDSS scores and ARRs, and this effect is significantly correlated with the erythrocyte concentrations of AZA metabolites, particularly 6-TGNs. A higher erythrocyte concentration of 6-TGNs is associated with better efficacy of AZA below the safe upper limits.

\section{Abbreviations \\ 6-MMPNs: 6-methylmercaptopurine nucleotides; 6-MP: 6-mercaptopurine; 6-TG: 6-thioguanine; 6-TGNs: 6-thioguanine nucleotides; ALL: Acute lymphoblastic leukemia; AQP4-IgG: Anti-aquaporin 4 antibodies; ARR: Annual relapse rates; AZA: Azathioprine; CNS: Central nervous system; \\ EDSS: Expanded disability status scale; HGPRT: Hypoxanthine-guanine phosphoribosyltransferase; HWE: Hardy-Weinberg equilibrium; MAF: Minor allele frequency; NMOSD: Neuromyelitis optica spectrum disorders; PDNS: Purine de novo synthesis; SD: Standard deviation; SLC28A: Solute carrier family 28; SLC29A: Solute carrier family 29; SNPs: Single nucleotide polymorphisms; TPMT: Thiopurine S-methyltransferase; TPNs: Thiopurine nucleotides}

\section{Acknowledgements}

Not applicable.

\section{Funding}

The design of the study and collection, analysis, and interpretation of data and in writing the manuscript were all supported by the Beijing Municipal Science \& Technology Commission (No. Z141107002514124).

\section{Availability of data and materials}

The datasets created during and analyzed during the current study available from the corresponding author on reasonable request.

\section{Authors' contributions}

XDL: study design, data analysis, manuscript writing and revising. SM: study design, azathioprine metabolites concentration detection and analysis. XG: study design, serum and CSF sample collection. HZ: patient's clinical information collection and follow-up. LY: azathioprine metabolites concentration detection. AZ: patient's clinical information collection and follow-up. YL: patient's clinical information collection and follow-up. XGL: Genetic variants and azathioprine metabolites concentration detection and analysis. ZZ: study design and manuscript revising. XZ: study design and manuscript revising. All authors read and approved the final manuscript.

\section{Ethics approval and consent to participate}

Written informed consent was obtained from all patients or their relatives, and this study was approved by the Ethics Committee of Beijing Tiantan Hospital Affiliated to Capital Medical University, Beijing, People's Republic of China (No. KY2015-031-02).

\section{Consent for publication}

All patients agreed with the publication.

\section{Competing interests}

The authors declare that they have no competing interests.

\section{Publisher's Note}

Springer Nature remains neutral with regard to jurisdictional claims in published maps and institutional affiliations.

\section{Author details}

${ }^{1}$ Neuroinfection and Neuroimmunology Center, Department of Neurology, Beijing Tiantan Hospital, Capital Medical University, 6 TiantanXili, Dongcheng District, Beijing 100050, People's Republic of China. ${ }^{2}$ China National Clinical Research Center for Neurological Diseases, Beijing Tiantan Hospital, Capital Medical University, 6 TiantanXili, Dongcheng District, Beijing 100050, People's Republic of China. ${ }^{3}$ Department of Pharmacy, Beijing Tiantan Hospital, Capital Medical University, 6 TiantanXili, Dongcheng District, Beijing 100050, People's Republic of China.

Received: 7 January 2017 Accepted: 22 June 2017

Published online: 05 July 2017

\section{References}

1. Wingerchuk DM, Banwell B, Bennett $J \mathrm{~L}$, Cabre P, Carroll W, Chitnis T, et al. International consensus diagnostic criteria for neuromyelitis optica spectrum disorders. Neurology. 2015;85:177-89.

2. Sellner J, Boggild M, Clanet M, Hintzen RQ, Illes Z, Montalban X, et al. EFNS guidelines on diagnosis and management of neuromyelitis optica. Eur J Neurol. 2010;17:1019-32.

3. Elsone L, Kitley J, Luppe S, Lythgoe D, Mutch K, Jacob S, et al. Long-term efficacy, tolerability and retention rate of azathioprine in 103 aquaporin-4 antibody-positive neuromyelitis optica spectrum disorder patients: a multicentre retrospective observational study from the UK. Mult Scler. 2014;20:1533-40. 
4. Mandler RN, Ahmed W, Dencoff JE. Devic's neuromyelitis optica: a prospective study of seven patients treated with prednisone and azathioprine. Neurology. 1998:51:1219-20

5. Costanzi C, Matiello M, Lucchinetti CF, Weinshenker BG, Pittock SJ, Mandrekar J, et al. Azathioprine: tolerability, efficacy, and predictors of benefit in neuromyelitis optica. Neurology. 2011;77:659-66.

6. Qiu W, Kermode AG, Li R, Dai Y, Wang Y, Wang J, et al. Azathioprine plus corticosteroid treatment in Chinese patients with neuromyelitis optica. J Clin Neurosci. 2015;22:1178-82.

7. Amin J, Huang B, Yoon J, Shih DQ. Update 2014: advances to optimize 6mercaptopurine and azathioprine to reduce toxicity and improve efficacy in the management of IBD. Inflamm Bowel Dis. 2015;21:445-52.

8. Cara CJ, Pena AS, Sans M, Rodrigo L, Guerrero-Esteo M, Hinojosa J, et al. Reviewing the mechanism of action of thiopurine drugs: towards a new paradigm in clinical practice. Med Sci Monit. 2004;10:Ra247-54.

9. Sahasranaman S, Howard D, Roy S. Clinical pharmacology and pharmacogenetics of thiopurines. Eur J Clin Pharmacol. 2008;64:753-67.

10. Fung C, Vaughn DJ, Mitra N, Ciosek SL, Vardhanabhuti S, Nathanson KL, et al. Chemotherapy refractory testicular germ cell tumor is associated with a variant in armadillo repeat gene deleted in Velco-cardio-facial syndrome (ARVCF). Front Endocrinol (Lausanne). 2012;3:163.

11. Fairchild CR, Maybaum J, Kennedy KA. Concurrent unilateral chromatid damage and DNA strand breakage in response to 6-thioguanine treatment. Biochem Pharmacol. 1986;35:3533-41.

12. Tiede I, Fritz G, Strand S, Poppe D, Dvorsky R, Strand D, et al. CD28dependent Rac1 activation is the molecular target of azathioprine in primary human CD4+ T Iymphocytes. J Clin Invest. 2003;111:1133-45.

13. Burnett HF, Tanoshima R, Chandranipapongse W, Madadi P, Ito S, Ungar WJ. Testing for thiopurine methyltransferase status for safe and effective thiopurine administration: a systematic review of clinical guidance documents. Pharmacogenomics J. 2014;14:493-502.

14. Salavaggione OE, Wang L, Wiepert M, Yee VC, Weinshilboum RM. Thiopurine S-methyltransferase pharmacogenetics: variant allele functional and comparative genomics. Pharmacogenet Genomics. 2005:15:801-15.

15. Collie-Duguid ES, Pritchard SC, Powrie RH, Sludden J, Collier DA, Li T, et al. The frequency and distribution of thiopurine methyltransferase alleles in Caucasian and Asian populations. Pharmacogenetics. 1999;9:37-42.

16. Umamaheswaran G, Kumar DK, Adithan C. Distribution of genetic polymorphisms of genes encoding drug metabolizing enzymes \& drug transporters - a review with Indian perspective. Indian J Med Res. 2014;139:27-65.

17. Gray JH, Owen RP, Giacomini KM. The concentrative nucleoside transporter family, SLC28. Pflugers Arch. 2004;447:728-34.

18. Errasti-Murugarren E, Molina-Arcas M, Casado FJ, Pastor-Anglada M. A splice variant of the SLC28A3 gene encodes a novel human concentrative nucleoside transporter-3 (hCNT3) protein localized in the endoplasmic reticulum. FASEB J. 2009;23:172-82.

19. Badagnani I, Chan W, Castro RA, Brett CM, Huang CC, Stryke D, et al. Functional analysis of genetic variants in the human concentrative nucleoside transporter 3 (CNT3; SLC28A3). Pharmacogenomics J. 2005:5:157-65.

20. Fotoohi AK, Lindqvist M, Peterson C, Albertioni F. Involvement of the concentrative nucleoside transporter 3 and equilibrative nucleoside transporter 2 in the resistance of T-lymphoblastic cell lines to thiopurines. Biochem Biophys Res Commun. 2006;343:208-15.

21. Mei S, Li X, Gong X, Li X, Yang L, Zhou H, Liu Y, Zhou A, Zhu L, Zhang X et al. LC-MS/MS analysis of erythrocyte thiopurine nucleotides and their association with genetic variants in patients with neuromyelitis optica spectrum disorders taking azathioprine. Ther Drug Monit 2016; doi: 10.1097/ FTD.0000000000000362.

22. Ketelslegers IA, Modderman PW, Vennegoor A, Killestein J, Hamann D, Hintzen $\mathrm{RQ}$. Antibodies against aquaporin-4 in neuromyelitis optica: distinction between recurrent and monophasic patients. Mult Scler. 2011;17:1527-30.

23. Rae W, Burke G, Pinto A. A study of the utility of azathioprine metabolite testing in myasthenia gravis. J Neuroimmunol. 2016;293:82-5.

24. Watanabe S, Misu T, Miyazawa I, Nakashima I, Shiga Y, Fujihara K, et al. Lowdose corticosteroids reduce relapses in neuromyelitis optica: a retrospective analysis. Mult Scler. 2007;13:968-74.

25. Swann PF, Waters TR, Moulton DC, Xu YZ, Zheng Q, Edwards M, et al. Role of postreplicative DNA mismatch repair in the cytotoxic action of thioguanine. Science (New York, NY). 1996;273:1109-11.

26. Elion GB. The purine path to chemotherapy. Science (New York, NY). 1989;244:41-7.
27. Jasiak-Zatonska M, Kalinowska-Lyszczarz A, Michalak S, Kozubski W. The immunology of Neuromyelitis Optica-current knowledge, clinical implications, controversies and future perspectives. Int J Mol Sci. 2016;17:273.

28. Lu HF, Shih MC, Chang YS, Chang JY, Ko YC, Chang SJ, et al. Molecular analysis of thiopurine S-methyltransferase alleles in Taiwan aborigines and Taiwanese. J Clin Pharm Ther. 2006:31:93-8.

29. Umamaheswaran G, Krishna Kumar D, Kayathiri D, Rajan S, Shewade DG, Dkhar SA, et al. Inter and intra-ethnic differences in the distribution of the molecular variants of TPMT, UGT1A1 and MDR1 genes in the south Indian population. Mol Biol Rep. 2012;39:6343-51.

30. Lowry PW, Franklin CL, Weaver AL, Pike MG, Mays DC, Tremaine WJ, et al. Measurement of thiopurine methyltransferase activity and azathioprine metabolites in patients with inflammatory bowel disease. Gut. 2001;49:665-70.

31. Fernandez-Calotti PX, Colomer D, Pastor-Anglada M. Translocation of nucleoside analogs across the plasma membrane in hematologic malignancies. Nucleosides Nucleotides Nucleic Acids. 2011;30:1324-40.

32. Molina-Arcas M, Marce S, Villamor N, Huber-Ruano I, Casado FJ, Bellosillo B, et al. Equilibrative nucleoside transporter-2 (hENT2) protein expression correlates with ex vivo sensitivity to fludarabine in chronic lymphocytic leukemia (CLL) cells. Leukemia. 2005;19:64-8.

33. Minuesa G, Purcet S, Erkizia I, Molina-Arcas M, Bofill M, Izquierdo-Useros N, et al. Expression and functionality of anti-human immunodeficiency virus and anticancer drug uptake transporters in immune cells. J Pharmacol Exp Ther. 2008;324:558-67.

\section{Submit your next manuscript to BioMed Central and we will help you at every step:}

- We accept pre-submission inquiries

- Our selector tool helps you to find the most relevant journal

- We provide round the clock customer support

- Convenient online submission

- Thorough peer review

- Inclusion in PubMed and all major indexing services

- Maximum visibility for your research

Submit your manuscript at www.biomedcentral.com/submit
) Biomed Central 